\title{
Multilayer Graphene Broadband Terahertz Modulators with Flexible Substrate
}

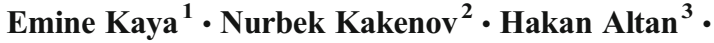 \\ Coskun Kocabas $^{2,4} \cdot$ Okan Esenturk $^{1}$ (D)
}

Received: 6 November 2017 / Accepted: 6 March 2018 /

Published online: 14 March 2018

(C) Springer Science+Business Media, LLC, part of Springer Nature 2018

\begin{abstract}
Fabrication of terahertz modulators as simple devices with high modulation depth across a broad bandwidth is still very challenging. In this study, four different chemical vapor deposition grown multilayer graphene (MLG) modulators based on MLG/ionic liquid/gold sandwich structures have been investigated. Flexible substrates (PVC and PE) were chosen as host materials, and devices were fabricated with three different thicknesses. The resultant MLG devices can be operated at low voltages between 0 and $3.4 \mathrm{~V}$ providing nearly complete modulation between 0.2 and $1.5 \mathrm{THz}$ with low insertion losses. Even with such low gate voltages, the devices have been doped significantly inducing 7-11-fold improvement in their sheet conductivities depending on device thickness. In addition, sheet conductivity has been improved more than three times as the graphene layer number increased from 30 to 100 . With the demonstration of promising device performances, the proposed modulators can be potential candidates for applications in terahertz and related optoelectronic technologies.
\end{abstract}

Keywords Attenuators $\cdot$ Filters $\cdot$ Terahertz optics $\cdot$ Integrated photonics

\section{Introduction}

Since its discovery in 2004, graphene has attracted intense attention in many fundamental areas due to its remarkable electronic and mechanical properties. With its gapless and symmetrical band structure, graphene has extraordinary physical properties such as a room-

Okan Esenturk

eokan@metu.edu.tr

1 Department of Chemistry, Middle East Technical University, 06800 Ankara, Turkey

2 Department of Physics, Bilkent University, 06800 Ankara, Turkey

3 Department of Physics, Middle East Technical University, 06800 Ankara, Turkey

4 UNAM-National Nanotechnology Research Center, Bilkent University, 06800 Ankara, Turkey 
temperature quantum Hall effect and micrometer long mean free path [1]. Its massless carriers result in an extremely high carrier mobility making graphene a unique material in applications of high-speed electronics. Single-layer graphene's fairly low (ca. 2.3\%) absorption of visible and IR radiation [2] makes it useful in the application of transparent electrodes, photodetectors, and broadband infrared electro-optical modulators [3]. Exploration of graphene carrier dynamics has shown that electronic structure of graphene is more sensitive to the terahertz region of the electromagnetic spectrum than to the IR and visible ranges [4], and terahertz beams allow characterization of carrier dynamics [5] near the Fermi level [6]. Therefore, graphene is recognized as a potentially active material for photosensitive terahertz devices in the application of active filters, switches, and modulators. These optical devices are urgently needed in order to advance in diverse applications such as nondestructive imaging, spectroscopy, biomedical diagnosis, ultrahigh wireless communication, and security.

Conventional terahertz modulators that are based on semiconductor materials [7] and hetero-structures containing a 2D electron gas [8] showed low modulation depths. Metal gates used in the structures can limit the working range of carrier density and Fermi energy tuning [9]. In addition, hybrid metasurfaces were also considered such as the one by Heyes et al. that achieved a broadband modulation between 0.5 and $1.5 \mathrm{THz}$ [10]. Compared to those firstgeneration devices, single layer graphene-based terahertz modulators have higher carrier mobilities with an electrically tunable carrier density and offer low insertion loss (0.2$0.5 \mathrm{~dB})[3,11]$. However, theoretically expected high modulation depth and broadband performance are difficult to achieve due to their strong dependence on quality of graphene [3] and unanticipated component effects such as substrate effects [2]. Improvement in terahertz modulation of single-layer graphene was achieved by integrating graphene with photonic cavities [12] or by metamaterials [13]. Kakenov et al. have demonstrated a flexible active terahertz surface constructed with a large-area single-layer graphene, a metallic reflective electrode, and an electrolytic medium in between. The device provides complete modulation in terahertz reflectivity at 2.8 . In addition, a $50 \%$ amplitude modulation at voltages between 20 and $+20 \mathrm{~V}$ is reported by Gao et al. using a gated single-layer graphene modulator with metallic ring aperture [13]. However, terahertz modulation in these studies is limited to a narrow bandwidth.

Increased modulation depth can be obtained by use of multilayer graphene (MLG) structure alone or MLG with ionic liquids [14-17]. Shen et al. presented 75\% modulation depth with a multilayer stack of alternating patterned graphene sheets in a metamaterial-based modulator [18]. However, narrowband operational range and polarization-dependent responses of metamaterial-based modulators may limit their future applications [11]. On the other hand, Baek et al. reported improved modulation when modulator layer numbers were increased from 1 to 12 [16].

In a study by Wu et al., the increase in modulation with increased number of graphene layers on electrodes was explained by elimination of boundary defects during multilayer formation in a graphene/ionic liquid/graphene device where ionic liquid forms interfaces with the graphene electrodes [15]. Kakenov et al. [19] presented an ionic liquid-based terahertz amplitude modulator where more than $50 \%$ modulation depth was obtained by efficient mutual gating of graphene electrodes and ionic liquid. Furthermore, Liu et al. achieved a modulation depth of $22 \%$ with a device utilizing ionic liquid. In addition, ionic liquid-based graphene modulators can demonstrate very high flexibility when used with flexible host materials such as polymers (i.e., polyvinyl chloride (PVC), polyethylene (PE), or polyethylene terephthalate (PET) ) [20]. Another advantage of MLG is that its optical response is dominated by layers that 
do not interact with substrate. Consequently, misleading optical results can be prevented since the dielectric substrates may cause a change in the Fermi level of a single layer graphene due to band gap opening [21].

A compromise between modulation depth, polarization dependence, ease of fabrication, design flexibility, large area production, and operational bandwidth exists in most of the studies reported in the literature. In this study, we present large-area MLG devices on flexible substrates that do not compromise on the modulation performance. The study experimentally demonstrated a remarkable performance on terahertz amplitude modulation by devices made from ionic liquid-doped MLG structures on polyvinyl chloride (PVC) and polyethylene (PE) substrates. The modulation depths were investigated at a broadband frequency range from 0.2 to $1.5 \mathrm{THz}$ with application of very low voltages ranging between 0 and $3.4 \mathrm{~V}$. The observed modulation range is limited by the spectrometer and appears frequency independent. The modulation is tunable and almost $100 \%$ attenuation is achieved at $3.4 \mathrm{~V}$. This performance is achieved by simple but effective structure of MLG terahertz modulators which enhanced gating effect of ionic liquid.

\section{Experimental}

A diagram of terahertz-TDS system used in these studies is shown in Fig. 1a. Coherent terahertz radiation was generated on a $<110>\mathrm{ZnTe}$ crystal via optical rectification by an amplified femtosecond laser ( $800 \mathrm{~nm}, 180 \mathrm{fs}, 1 \mathrm{kHz}$ ). The radiation is detected from retarded phase of the detection pulse by the oscillating terahertz field in a second ZnTe crystal, and polarization change is monitored by a balanced photodiode with a quarter-wave plate and a Wollaston prism. The photodiode voltage is detected using a lock-in amplifier. The system is enclosed in a dry air-purged box to minimize water vapor attenuation of terahertz. The spectrometer effective working range is 0.2 to $1.5 \mathrm{THz}$ with the MLG devices.

Multilayer graphene samples were grown on nickel foils using chemical vapor deposition method. Due to high solubility of carbon atoms on Ni, highly crystalline MLGs with varying layer numbers were grown. The growth process takes place in quartz chamber under the

(a)

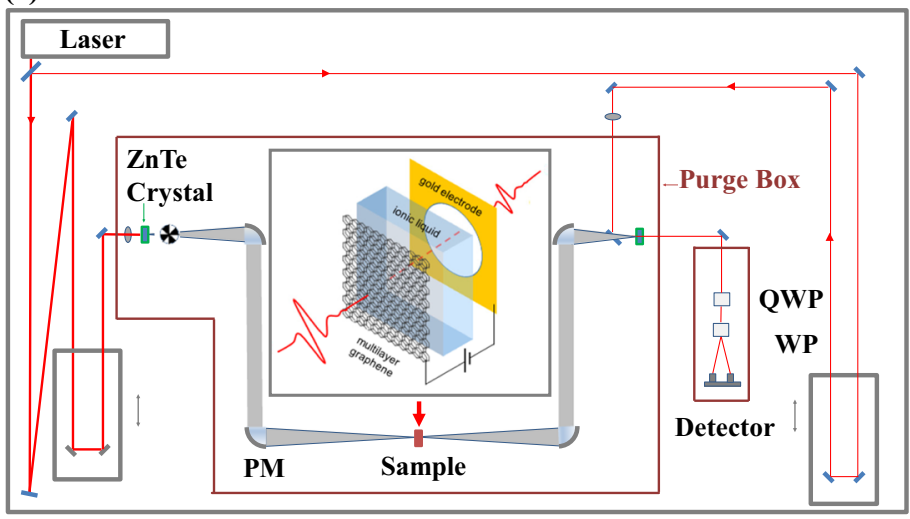

(b)

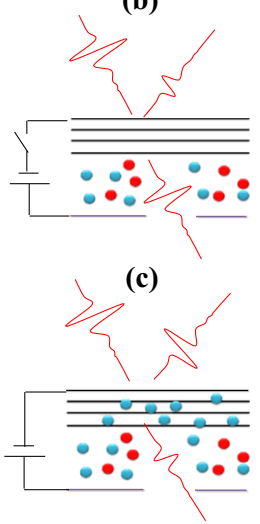

Fig. 1 (a) Terahertz setup and MLG structure (inset) consisting of MLG sandwiched between host (PVC or PE), electrolyte, and gold electrode. Drawings show (b) no doping case at $0 \mathrm{~V}$ and (c) intercalation of ions through the graphene 
presence of argon, hydrogen, and methane gases. The temperature in the chamber determines the layer number of graphene samples. The samples were grown at 850,900 , and $1000{ }^{\circ} \mathrm{C}$ corresponding to nearly 30, 60, and 100 layers, respectively, estimated from optical measurements [22]. After the growth, MLG samples were transferred on PVC (labeled as MLG850, MLG900, and MLG1000) or on PE (MLG1000PE) by lamination, and nickel was removed with iron chloride $\left(\mathrm{FeCl}_{3} \cdot 6 \mathrm{H}_{2} \mathrm{O}\right)$ solution.

The inset of Fig.1a illustrates the fabricated MLG structure. The device consists of MLG on substrate (PE or PVC) and gold electrodes sandwiching an ionic liquid, Diethylmethyl(2methoxyethyl)ammoniumbis (trifluoro-methylsulfonyl) imide. Separator, $35 \mu \mathrm{m}$ Whatman tissue, is used to keep ionic liquid and to isolate MLG and gold electrode from contacting. The gate electrode, 80 -nm gold on substrate, has a 5-mm circular opening for terahertz transmission. Eighty-nanometer gold is sputtered with a mask for electrical contact, and wires are bonded to sputtered gold and to gold electrode. The separation, tissue, between MLG and gold-gating electrode prevents leaking and enables intercalation. At zero bias, the transmittance of MLG devices were maximum suggesting very low doping level (Fig. 1b). Upon application of bias voltage, the ions of the same polarity intercalate through layers of graphene $[22,23]$ resulting in attenuation of terahertz transmission (Fig. 1c). Recovery is slow, and application of a gate voltage beyond $3.5 \mathrm{~V}$ may damage the device.

\section{Results and Discussion}

Figure 2a presents changes in terahertz waveforms for the MLG850 sample as the applied voltage was varied. The corresponding frequency domain data of the waveforms are given in Fig. 2b. The data show no observed changes in phase, a result that might be due to graphene's robust nature under electrolyte gating [15] and/or its much smaller thickness compared to the terahertz wavelength. Transmission spectra of MLG devices relative to $0 \mathrm{~V}$ are given in Fig. $2 \mathrm{c}-\mathrm{f}$. At applied voltages up to $1.5 \mathrm{~V}$, less than $20 \%$ modulation was observed in all devices. A modulation between 20 and $30 \%$ at ca. $2 \mathrm{~V}$ was achieved with greater MLG thickness. The MLG devices had more than $80 \%$ modulation at $2.6 \mathrm{~V}$, except MLG850 which had modulation around $50 \%$. Almost full power modulation was achieved with all MLG devices at voltages beyond $3 \mathrm{~V}$. The modulation depth is significantly improved compared to single [4] and multilayer [16] devices over a very broad range. The modulation was fairly flat and featureless over a broad frequency range especially at low voltages suggesting that modulation is frequency-independent. Compared to other devices, the thinnest unit, MLG850, provided a more controllable modulation with the applied voltage. Features such as the ones around $1.2 \mathrm{THz}$ in Fig. 2b, c at low voltages (which are higher than $100 \%$ at $0.5 \mathrm{~V}$ ) are possibly artifacts due to signal-to-noise limits and transmittance calculations relative to $0 \mathrm{~V}$. Otherwise, such features are expected to become stronger as the voltage or attenuation is increased.

Figure 3a presents the change in terahertz peak amplitude of all four devices at voltages between 0 and $3.4 \mathrm{~V}$. The terahertz peak amplitude results from an interference of all frequencies in the field. Therefore, it represents an average behavior. The maximum transmission was observed at $0 \mathrm{~V}$ while the minimum was reached beyond $3 \mathrm{~V}$. As the gate voltage was increased, an almost linear decrease in amplitude was observed up to ca. $2 \mathrm{~V}$. Transmission decreased sharply as the gate voltage was increased from 2.0 to $2.8 \mathrm{~V}$. At gate voltages above ca. $2.8 \mathrm{~V}$, the transmission remained relatively flat (up to a maximum applied voltage of $3.4 \mathrm{~V}$ ). The observed change in terahertz amplitude was provided with voltage-controlled 

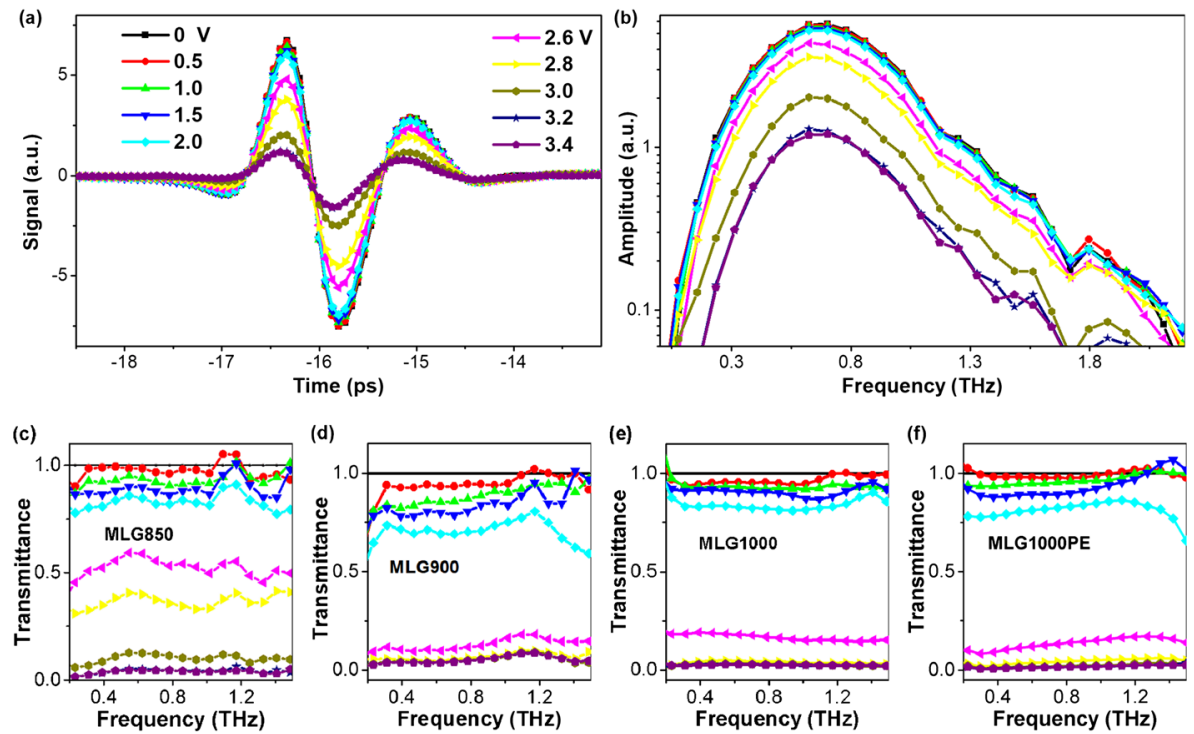

Fig. 2 (a) Transmitted terahertz waveforms as voltage is varied on the device, MLG850. (b) Corresponding frequency domain amplitudes. Terahertz transmittance of (c) MLG850, (d) MLG900, (e) MLG1000, and (f) MLG1000PE at all applied voltages relative to $0 \mathrm{~V}$

changes in mobile carrier density. The voltage-dependent behavior of all MLG devices was very similar except the thinnest device (MLG850) where steep decrease started at slightly higher voltage. Four probe measurements of the voltage-dependent sheet resistance behavior of MLG850 is provided in inset of Fig. 3a and shows a very similar behavior. The comparison of the peak amplitude behavior with the sheet resistance and with the behaviors at selected voltages (Fig. 3b) suggest that observed modulation with the set voltage appears to be independent of the terahertz frequencies and is limited only by the instrument response.
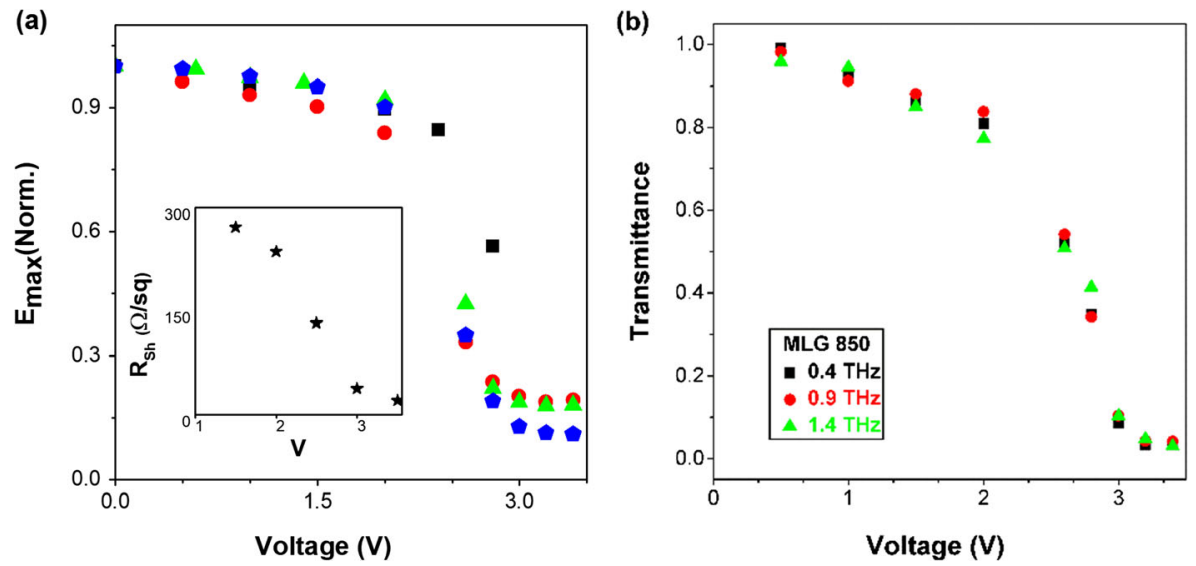

Fig. 3 (a) Transmission modulation with voltage at terahertz peak amplitude (MLG850 black square; MLG900 red circle; MLG1000 green triangle; MLG1000PE blue pentagon). (inset: voltage-dependent sheet resistance of MLG850 measured by four probe). (b) Transmittance of MLG850 device for three selected frequencies of 0.4 , 0.9 , and $1.4 \mathrm{THz}$ 
Optical conductivity of graphene appears to follow its electrical conductivity at the sampled terahertz frequencies and is described by the Drude model $[6,24]$. The imaginary part of the conductivity was neglected when applying the model since no significant phase shift in the time domain (Fig. 2a) was observed. All changes observed in conductivity are expected to result from a change in carrier density and/or change in scattering time [25]. Therefore, terahertz sheet conductivities $\left(\sigma_{s h}\right)$ of MLG devices are proportional to the amplitude ratio of reference (PVC or PE) substrate to MLG sample as given in Eq. 1 [26]:

$$
\sigma_{s h}=(n+1)\left(\frac{A_{\text {subsrate }}}{A_{M L G}}-1\right) / Z_{0}
$$

Here, $Z_{0}$ is free-space impedance, and $n$ is substrate refractive index. Frequency-dependent sheet conductivity of MLG850 at $0 \mathrm{~V}$ is given in Fig. 4a as an example. The behaviors of others were similar to the thinnest device. At the Dirac point, all devices showed featureless sheet conductivities, and their DC conductivities were estimated to be 4.4, 7.5, 17.8, and $10.3 \mathrm{mS}$ for MLG850, MLG900, MLG1000, and MLG1000PE, respectively.

The DC conductivity of the device having a PE substrate (MLG1000PE) appeared to be lower than its PVC counterpart. This difference was most likely due to differences in the quality of the graphene [26] since it affects the carrier scattering time. These DC conductivities are close to the ones determined from four probe measurements (3.3 mS for MLG850, $5.8 \mathrm{mS}$ for MLG900, $31.2 \mathrm{mS}$ for MLG1000) and are consistent with those reported for the similar devices $[11,16,26]$. Carrier densities were estimated as ca. $1.5 \times 10^{12} \mathrm{~cm}^{-2}$ for MLG850, ca. $4.5 \times 10^{12} \mathrm{~cm}^{-2}$ for MLG900, and ca. $1.3 \times 10^{14} \mathrm{~cm}^{-2}$ for MLG1000 using experimental sheet
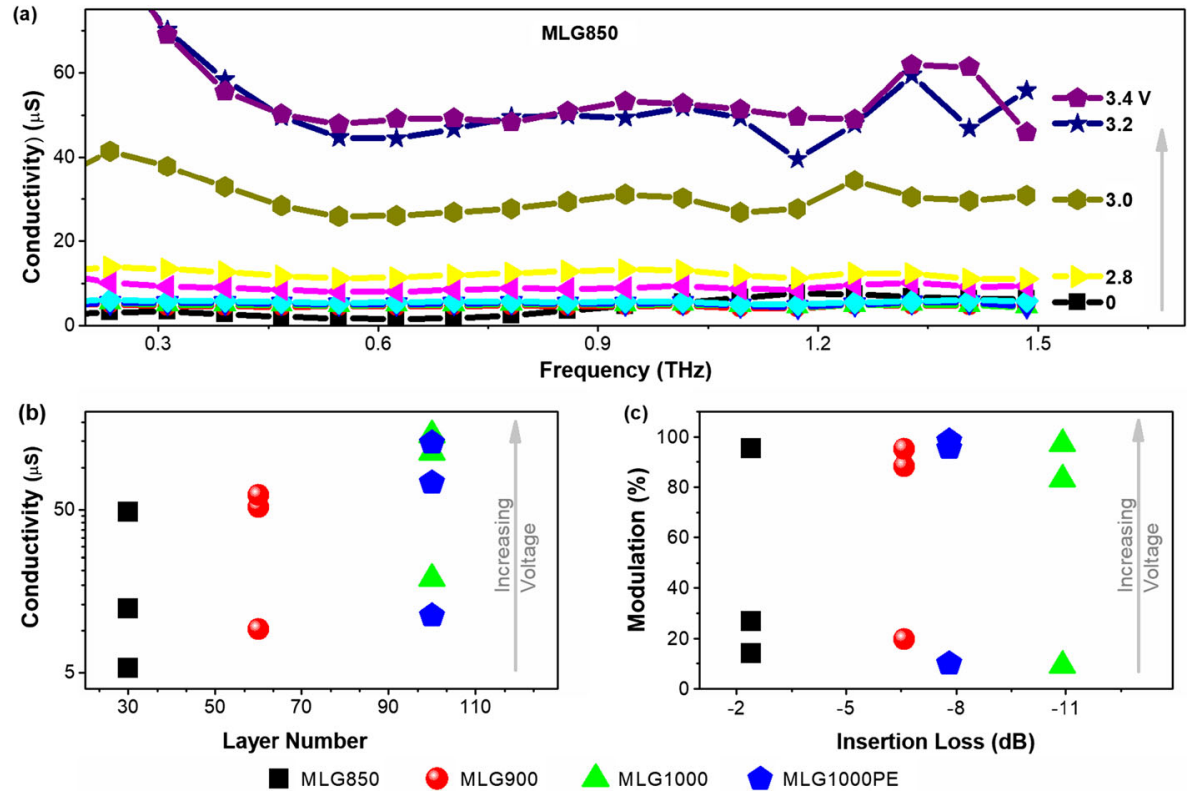

Fig. 4 (a) Frequency-dependent terahertz sheet conductivities of MLG850. Trends in (b) terahertz sheet conductivities and (c) modulation at selected frequency of $0.8 \mathrm{THz}$ for selected voltages of $1.5,2.8$, and $3.4 \mathrm{~V}$ for clarity. The $\mathrm{x}$ scale in Fig. $4 \mathrm{c}$ is the insertion loss of MLG devices (initial attenuation of the device when it is first introduced into the optical path) 
resistance and an average scattering time of $200 \mathrm{fs}$ [27]. Sheet conductivities $\left(\sigma_{s h}\right)$ at applied voltages were calculated using Eq. 2 [28], which is derived from Eq. 1:

$$
\sigma_{s h}=\frac{n+1}{Z_{0}}\left[\frac{A_{0}}{A_{\text {gate }}}\left(1+\sigma_{s h, 0} \frac{Z_{0}}{n+1}\right)-1\right]
$$

where $\mathrm{A}_{0}$ and $\mathrm{A}_{\text {gate }}$ are the transmitted terahertz field amplitudes of the devices at $0 \mathrm{~V}$ and gate voltages, respectively. The results are reported in Fig. 4a for MLG850. Figure $4 \mathrm{~b}$ presents conductivities of all devices at representative voltages of $1.5,2.8$, and $3.4 \mathrm{~V}$ for $0.8 \mathrm{THz}$. Conductivities increased significantly with doping, and percent increase at $3.4 \mathrm{~V}$ relative to $0 \mathrm{~V}\left[\Delta \sigma=\left(\sigma_{3.4}-\sigma_{0}\right) / \sigma_{0}\right]$ was ca. $1000 \%$ for MLG850, 715\% for MLG900, 705\% for MLG1000, and $1150 \%$ for MLG1000PE. A similar high increase is explained by an increase in hole carrier concentration in a study by Qi et al. [25]. Besides the doping effect, graphene thickness can also affect the conductivity significantly. When the thinnest (ca. 30 layers) and the thickest (ca. 100 layers) devices were compared at $0 \mathrm{~V}\left[\Delta \sigma=\left(\sigma_{100}-\sigma_{30}\right) / \sigma_{30}\right]$, an enhancement of ca. $300 \%$ was observed. The difference was still significant but less pronounced (197\%) at $3.4 \mathrm{~V}$ for the same devices. The increase in layer numbers should not affect terahertz conductivity of MLG devices or their scattering time [25] since the sheet conductivity

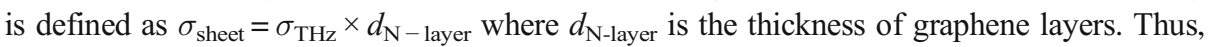
only the sheet conductivity should increase due to increased carrier density with thickness. Similarly, an increase of $73 \%$ in the sheet conductivity was noted as the graphene layer number was increased from 1 to 12 in a study by Baek et al. In addition, Wu et al. had also shown an increased sheet conductivity with an increase in layer number [17].

In addition to being a preferential host material for flexible photonic devices, PVC and PE are also preferred for their very low insertion losses of less than $1 \mathrm{~dB}$ at all frequencies, which is much less than that of the devices. The thinnest device had an average of $3 \mathrm{~dB}$ insertion loss, and the loss was increased with the layer thickness. Thus, the highest loss was observed for the thickest devices as ca. $8 \mathrm{~dB}$ for MLG1000PE and $11 \mathrm{~dB}$ for MLG1000. Figure 4c presents modulation of terahertz amplitude versus the insertion loss at $0.8 \mathrm{THz}$ for selected voltages of 1.5, 2.8, and 3.4 V. Here, the insertion loss is the initial loss of the terahertz power when the device is inserted in the beam path and modulation represents the further change in the transmission of the terahertz wave. Any initial attenuation of the terahertz field by the ionic liquid prior to applied voltage is also included in the insertion losses. The best performance was achieved with the thinnest MLG device with its much lower insertion loss and almost $100 \%$ modulation depth at $3.4 \mathrm{~V}$. Depending on the application type, devices with optimal numbers of graphene layers can be designed considering the trade-off between the conductivity, modulation, and insertion loss at the preferred voltage.

\section{Conclusions}

In this study, broadband modulation behavior of four different terahertz devices based on multilayer graphene was investigated by terahertz time-domain spectroscopy. The thinnest device with ca. 30 layers provided almost complete modulation of terahertz waves at an operation voltage of less than $3.4 \mathrm{~V}$. The effect of increased layer number on terahertz modulation was also investigated, and near-complete modulation was demonstrated at all thicknesses. However, as the layer thickness increased, an increase in the insertion loss was 
also observed. With the strong gating effect of dopant molecules, it was possible to achieve a tunable modulation in terahertz transmission between 0.2 and $1.5 \mathrm{THz}$ as a broadband response at very low operation voltages, 0 to $3.4 \mathrm{~V}$. Here, the modulation is frequencyindependent and bandwidth appears to be limited only by the instrument. Even at such low gate voltages, the devices were doped significantly inducing an enormous improvement in their sheet conductivities. Sheet conductivities of the devices were also improved with increasing thickness of the active layer. Such controlled and targeted behaviors are promising for new terahertz devices for desired conductivities and modulations. This study along with the previously reported studies have shown that ionic liquid-integrated MLG devices are a promising platform to produce terahertz active filters with controlled modulation and are expected to have a strong impact on terahertz optoelectronic devices in the near future in many application areas.

Funding Information This research was supported by Türkiye Bilimsel ve Teknolojik Arastirma Kurumu-TUBITAK. (Scientific and Technological Research Council of Turkey). E.K. and O.E. acknowledge funding from TUBITAK grant 111T393. N.K., H.A., and C.K. acknowledge funding from TUBITAK grant $114 \mathrm{~F} 379$.

\section{References}

1. K. S. Novoselov, A. K. Geim, S. V. Morozov, D. Jiang, Y. Zhang, S. V. Dubonos, I. V. Grigorieva, and A. A. Firsov, Science. 306, 666 (2004).

2. B. Sensale-Rodriguez, R. Yan, M. M. Kelly, T. Fang, K. Tahy, W. S. Hwang, D. Jena, L. Liu, and H. G. Xing, Nat. Commun. 3, 780 (2012).

3. B. Sensale-Rodriguez, R. Yan, S. Rafique, M. Zhu, W. Li, X. Liang, D. Gundlach, V. Protasenko, M. M. Kelly, D. Jena, L. Liu, and H. G. Xing, Nano Lett. 12, 4518 (2012).

4. I. Maeng, S. Lim, S. J. Chae, Y. H. Lee, H. Choi, and J.-H. Son, Nano Lett. 12, 551 (2012).

5. J. Lloyd-Hughes and T.-I. Jeon, J. Infrared, Millimeter, Terahertz Waves 33, 871 (2012).

6. L. Ren, Q. Zhang, J. Yao, Z. Sun, R. Kaneko, Z. Yan, S. Nanot, Z. Jin, I. Kawayama, M. Tonouchi, J. M. Tour, and J. Kono, Nano Lett. 12, 3711 (2012).

7. H. T. Chen, W. J. Padilla, J. M. O. Zide, A. C. Gossard, A. J. Taylor, and R. D. Averitt, Nature 444, 597 (2006).

8. T. Kleine-Ostmann, K. Pierz, G. Hein, P. Dawson, M. Marso, and M. Koch, J. Appl. Phys. 105, 93707 (2009).

9. A. Locatelli, G. E. Town, and C. De Angelis, IEEE Trans. Terahertz Sci. Technol. 5, 351 (2015).

10. J. E. Heyes, W. Withayachumnankul, N. K. Grady, D. R. Chowdhury, A. K. Azad, and H.-T. Chen, Appl. Phys. Lett. 105, 181108 (2014).

11. B. Sensale-Rodriguez, T. Fang, R. Yan, M. M. Kelly, D. Jena, L. Liu, and H.(Grace) Xing, Appl. Phys. Lett. 99, 113104 (2011).

12. N. Kakenov, O. Balci, T. Takan, V. A. Ozkan, H. Altan, and C. Kocabas, ACS Photonics 3, 1531 (2016).

13. W. Gao, J. Shu, K. Reichel, D. V. Nickel, X. He, G. Shi, R. Vajtai, P. M. Ajayan, J. Kono, D. M. Mittleman, and Q. Xu, Nano Lett. 14, 1242 (2014).

14. F. Shi, Y. Chen, P. Han, and P. Tassin, Small 11, 6044 (2015).

15. Y. Wu, C. La-O-Vorakiat, X. Qiu, J. Liu, P. Deorani, K. Banerjee, J. Son, Y. Chen, E. E. M. Chia, and H. Yang, Adv. Mater. 27, 1874 (2015).

16. I. H. Baek, K. J. Ahn, B. J. Kang, S. Bae, B. H. Hong, D. I. Yeom, K. Lee, Y. U. Jeong, and F. Rotermund, Appl. Phys. Lett. 102, 191109 (2013).

17. Y. Wu, X. Ruan, C.-H. Chen, Y. J. Shin, Y. Lee, J. Niu, J. Liu, Y. Chen, K.-L. Yang, X. Zhang, J.-H. Ahn, and H. Yang, Opt. Express 21, 21395 (2013).

18. N. H. Shen, P. Tassin, T. Koschny, and C. M. Soukoulis, Phys. Rev. B - Condens. Matter Mater. Phys. 90, 115437 (2014).

19. N. Kakenov, O. Balci, E. O. Polat, H. Altan, and C. Kocabas, J. Opt. Soc. Am. B 32, 1861 (2015).

20. J. Liu, P. Li, Y. Chen, X. Song, M. Qi, W. Yang, Q. Fei, B. Zheng, H. Jiarui, H. Yang, Q. Wen, and W. Zhang, Opt. Lett. 41, 816 (2016).

21. J. Y. Kim, C. Lee, S. Bae, K. S. Kim, B. H. Hong, and E. J. Choi, Appl. Phys. Lett. 98, 201907 (2011). 
22. E. O. Polat, O. Balc1, and C. Kocabas, Sci. Rep. 4, 6484 (2014).

23. E. O. Polat, H. B. Uzlu, O. Balci, N. Kakenov, E. Kovalska, and C. Kocabas, ACS Photonics 3, 964 (2016).

24. A. J. Frenzel, C. H. Lui, W. Fang, N. L. Nair, P. K. Herring, P. Jarillo-Herrero, J. Kong, and N. Gedik, Appl. Phys. Lett. 102, 113111 (2013).

25. M. Qi, Y. Zhou, F. Hu, X. Xu, W. Li, A. Li, J. Bai, and Z. Ren, J. Phys. Chem. C 118, 15054 (2014).

26. Y. Zhou, X. Xu, F. Hu, X. Zheng, W. Li, P. Zhao, J. Bai, and Z. Ren, Appl. Phys. Lett. 104, 51106 (2014).

27. M. Orlita, C. Faugeras, P. Plochocka, P. Neugebauer, G. Martinez, D. K. Maude, A.-L. Barra, M. Sprinkle, C. Berger, W. A. de Heer, and M. Potemski, Phys. Rev. Lett. 101, 267601 (2008).

28. M. Nagel, A. Safiei, S. Sawallich, C. Matheisen, T. M. Pletzer, A. A. Mewe, N. van der Borg, I. Cesar, and H. Kurz, Proc. 28th Eur. Photovolt. Sol. Energy Conf. Exhib. 856 (2013). 